\title{
Eu realizo mais atividades burocráticas: análise da Assistência Farmacêutica na Atenção Primária a Saúde
}

\author{
Indara Cavalcante Bezerra (iD \\ Universidade Estadual do Ceará - UECE - Fortaleza (CE) - Brasil
}

Raimunda Magalhães da Silva (D)

Universidade de Fortaleza - UNIFOR - Fortaleza (CE) - Brasil

Jonas Loiola Gonçalves (iD

Universidade de Fortaleza - UNIFOR - Fortaleza (CE) - Brasil

Maria Salete Bessa Jorge (D)

Universidade Estadual do Ceará - UECE - Fortaleza (CE) - Brasil

Cleoneide Paulo Oliveira Pinheiro (D)

Centro Universitário Estácio de Sá (Estácio/FIC) - Fortaleza (CE) - Brasil

Francisco Antônio da Cruz Mendonça (iD

Centro Universitário Estácio de Sá (Estácio/FIC) - Fortaleza (CE) - Brasil

\section{RESUMO}

Objetivo: Apreender a percepção dos farmacêuticos acerca da Assistência Farmacêutica (AF) na Atenção Primária à Saúde (APS) com ênfase nas pesquisas e ações realizadas. Métodos: Realizaram-se entrevistas em profundidade com sete farmacêuticos pesquisadores, gerentes e profissionais vinculados à AF do município de Fortaleza, em 2015, utilizando-se processo avaliativo em perspectiva múltipla, plural e construtivista, com o propósito de utilizar os resultados das pesquisas para incorporá-las à práxis farmacêutica. Resultados: As práticas do farmacêutico na APS são pontuais e justificadas sob o ponto de vista da legislação que exige a presença do profissional para a dispensação de medicamentos de controle especial. Também, o modelo praticado no município de Fortaleza, pautado na terceirização do vínculo empregatício e da gestão da farmácia, tem provocado o isolamento da AF aos demais serviços e setores da APS, favorecendo a quebra da integralidade do cuidado e a perdas significativas na resolubilidade. Conclusão: O fluxo das pesquisas está interrompido, fragmentado, confuso e burocrático, o que sugere um melhor gerenciamento das pesquisas, propondo um fluxo efetivo e contínuo que tenha origem na real demanda da comunidade e cujos resultados sejam direcionados para a construção de novas estratégias que otimizem os recursos, as decisões e as práticas em saúde.

Descritores: Assistência Farmacêutica; Gestão em Saúde; Atenção Primária à Saúde; Avaliação em Saúde; Avaliação da Pesquisa em Saúde.

\section{INTRODUÇÃO}

A Atenção Primária à Saúde (APS) corresponde a uma estratégia de organização voltada para responder de forma regionalizada, contínua e sistematizada à maior parte das necessidades de saúde de uma população, integrando ações preventivas e curativas, bem como a atenção a indivíduos e comunidades. No Brasil, é desenvolvida de forma descentralizada, capilarizada e próxima da vida das pessoas. Seu locus deve ser o contato preferencial dos usuários, a principal porta de entrada e o centro de comunicação com toda a Rede de Atenção à Saúde, uma vez que se orienta pelos princípios da universalidade, da acessibilidade, do vínculo, da continuidade do cuidado, da integralidade da atenção, da responsabilização, da humanização, da equidade e da participação social(1).

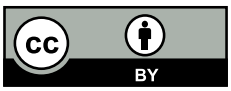


A avaliação de programas e serviços sociais em saúde é primordial para o aprimoramento do ciclo de desenvolvimento das políticas públicas, principalmente no tocante à gestão e planejamento de suas ações e está associada ao ideário de manutenção de qualidade dos serviços oferecidos pelo Estado(2). No processo avaliativo, a opinião dos cidadãos também é considerada ${ }^{(1)}$, entretanto, essa ferramenta de avaliação, por vezes, não contempla a interface da APS com outras políticas e áreas, a exemplo da Assistência Farmacêutica (AF).

Historicamente, a partir da descentralização administrativa das unidades de saúde estaduais e federais para o âmbito municipal, os serviços farmacêuticos acompanharam a lógica da territorialização e passaram a ser exercidos, em sua maioria, nos municípios. Esse processo estimulou iniciativas estaduais para a estruturação da assistência farmacêutica na APS, com a organização de programas no seu território ${ }^{(3)}$. Mesmo com tais avanços, problemas podem persistir e comprometer a missão de garantir o acesso da população aos medicamentos e a racionalidade do uso, uma vez que o serviço farmacêutico ainda opera de acordo com o modelo curativo, de tal forma que a farmácia atende quase que exclusivamente à essas demandas, prejudicando a atividade de atenção farmacêutica de integração com a equipe ${ }^{(3)}$.

Desse modo, a participação ativa do farmacêutico nas equipes multiprofissionais é vista como necessidade para o redesenho do modelo de atenção às condições crônicas e para a melhoria dos resultados em saúde, particularmente no nível dos cuidados primários ${ }^{(4)}$.

As avaliações no âmbito da AF no Brasil, realizadas na lógica da tríade de Donabedian (estrutura-processoresultado), têm recebido investimentos e vêm demonstrando resultados isolados, às vezes, por levarem em consideração somente aspectos relacionados à estrutura ou ao processo; poucas vezes são realizadas avaliações que consideram os resultados da AF. A estrutura, o processo e o resultado podem não ter uma conexão direta e gerar um juízo de valor na avaliação da AF sem muitos subsídios, pois, em alguns serviços, a falta de estrutura dificulta o alcance dos resultados ou limita o desenvolvimento dos processos. Uma excelente estrutura ou processos adequados também não garantem o avanço de resultados positivos na $\mathrm{AF}^{(5)}$.

Nesse sentido, uma avaliação importante foi realizada pelo Departamento de Assistência Farmacêutica do Ministério da Saúde brasileiro (DAF/MS), em conjunto com a Organização Pan-Americana de Saúde (OPAS), entre os anos de 2003/2004, intitulada "Avaliação da Assistência Farmacêutica no Brasil: Estrutura, processo e resultados" (6). Esse estudo avaliou os resultados das políticas farmacêuticas por meio do acesso, da qualidade e do uso racional de medicamentos. O resultado permitiu visualizar um panorama da situação da AF, o que contribuiu para a construção de estratégias de intervenção e estabelecimento de um modelo de monitoramento da política de medicamentos no Brasil, subsidiando a Política Nacional da Assistência Farmacêutica (PNAF) ${ }^{(5)}$, atestando a importância das pesquisas quando orientadas para a aplicabilidade de seus resultados.

No cenário internacional, a AF tem avançado ainda mais, especialmente em países europeus, onde os serviços de APS contam com a presença do "farmacêutico comunitário", cujas ações estão voltadas, não apenas para a aquisição de insumos e medicamentos, mas para o assessoramento e revisão de prescrições junto aos médicos e para a orientação farmacoterapêutica adequada aos usuários ${ }^{(7-15)}$.

No Brasil, ao analisar a avaliação de pesquisas sobre AF e sua interlocução com a APS, percebe-se 'um atraso' quando comparado ao cenário europeu explicitado, revelando uma lacuna do conhecimento importante nessa área. Em visita a Biblioteca Virtual em Saúde (BVS) e à Biblioteca Virtual Portal de Periódicos CAPES/MEC, para conhecer o cenário de pesquisas realizadas no Brasil na última década quanto à avaliação da AF na APS entre os, foram encontrados apenas quatro trabalhos ${ }^{(16-19)}$.

Observa-se, pois, que a avaliação de serviços e programas sociais ainda é incipiente, o que suscita mais estudos para esse foco. Os resultados dos trabalhos apontam para uma realidade reducionista da profissão farmacêutica, visto que na interface da APS com a AF, as ações continuam engessadas no ciclo logístico do medicamento, ao seu consumo e acesso. Portanto, essas pesquisas revelam o entendimento limitado da avaliação da AF voltado para ações de gestão de insumos, o que reduz o potencial das ações desses profissionais em contribuir no cuidado à APS. Com base nesse contexto, o presente trabalho objetivou apreender a percepção dos farmacêuticos acerca da Assistência Farmacêutica na Atenção Primária à Saúde com ênfase nas pesquisas e ações realizadas.

\section{MÉTODOS}

Este estudo faz parte de um projeto intitulado "Avaliação das pesquisas sobre assistência farmacêutica na atenção primária à saúde e sua aplicabilidade no SUS: olhares analisadores da realidade". O objeto de investigação aproxima a opção teórico-metodológica para o campo das abordagens qualitativas, realizando um processo avaliativo numa perspectiva múltipla, plural e construtivista, com o propósito de utilizar os resultados para incorporar 
à práxis farmacêutica(20-22). Nesse sentido, buscou-se compreender, através da opinião dos farmacêuticos, como as pesquisas sobre a Assistência Farmacêutica repercutem nos serviços de Atenção primária à Saúde possibilitando a compreensão dessas relações no contexto político e social do SUS.

Para tanto, a avaliação caminhou diante da fusão metodológica hermenêutica-dialética, cuja hermenêutica se move como: compreensão, liberdade, necessidade, força, consciência histórica, todo e partes, como categorias filosóficas fundantes de significado, enquanto que a dialética articula as ideias de crítica, movimento e transformação da natureza e da realidade social(23,24). Com efeito, essa junção de métodos, sob a ótica do pensamento, faz a síntese dos processos compreensivos e $\operatorname{críticos}^{(24)}$, pois a mesma razão que compreende, esclarece e reúne, também contesta, dissocia e critica ${ }^{(23)}$.

Inicialmente realizou-se o levantamento de todos os projetos, cujo objeto de estudo estivesse relacionado à Assistência Farmacêutica, realizadas no município de Fortaleza, Ceará, através do banco de registros de projetos e pesquisas cadastrados pela Coordenadoria de Gestão do Trabalho e Educação em Saúde (COGTES). O propósito dessa etapa foi identificar os objetivos, resultados e considerações finais das pesquisas realizadas.

A pesquisa em campo foi desenvolvida no município de Fortaleza, Ceará, cuja Rede de Atenção à Saúde (RAS) está dividida em sete regiões administrativas chamadas de Secretarias Executivas Regionais (SER). A escolha dos participantes e do locus da pesquisa ocorreu por indicação de farmacêuticos da própria RAS no campo estudado, por conhecerem suas práticas e entenderem que os sujeitos indicados poderiam contribuir para as questões essenciais da investigação.

Realizaram-se entrevistas em profundidade com sete farmacêuticos, nas funções de pesquisadores, gerentes e profissionais vinculados à Assistência Farmacêutica do município de Fortaleza há pelo menos um ano. A coleta das informações ocorreu em 2015 nos seguintes locais: Unidade de Atenção Primária à Saúde (UAPS) Rigoberto Romero na SER I; UAPS Anastácio Magalhães na SER III; UAPS Galba Araújo na SER V; na Célula de Assistência Farmacêutica do Município de Fortaleza profissional e no Departamento de Farmácia da Universidade Federal do Ceará.

Ressalta-se que a organização e análise dos dados foram tratados diante da combinação metodológica hermenêutica-dialética, seguindo os pressupostos teóricos de Habermas ${ }^{(23,25,26)}$. Essa perspectiva crítica e reflexiva, foca na linguagem de maneira a considerá-la quanto à constituição dos sujeitos, à produção dos sentidos e à sua relação com o mundo, possibilitando o entendimento universo de significados ${ }^{(23,24)}$.

Os resultados apresentados preservam a identidade dos participantes e utiliza o discurso coletivo por entender que as falas dos farmacêuticos representam essa classe profissional. Os participantes assinaram o Termo de Consentimento Livre e Esclarecido e o projeto de pesquisa foi aprovado por Comitê de Ética em Pesquisa com Seres Humanos, sob Parecer $n^{\circ} 662.093$.

\section{RESULTADOS}

O presente trabalho acessou os projetos e pesquisas no âmbito da AF na APS em Fortaleza e no Ceará, a partir dos arquivos da COGTES, no intuito de entender o fluxo dessas pesquisas e observar seu direcionamento aos gestores, além de apreender sobre seus resultado e aplicabilidade.

A busca inicial se deteve aos arquivos correspondentes aos anos 2013 e 2014 e procurou por aqueles que abordassem a temática da AF. Dentre os 428 projetos cadastrados nesse período, foram encontrados 4 projetos com a temática da AF, e ainda assim estes não apresentavam relação com a APS para configurarem-se como objeto deste estudo. Além disso, outras pesquisas que foram desenvolvidas com essa temática e realizadas nos serviços de saúde pública em Fortaleza e que já haviam sido publicadas nas bibliotecas virtuais das universidades e nas revistas científicas, não foram encontradas nos registros da COGTES, sugerindo que há uma falha no protocolamento desses projetos, na organização dos registros e provável subnotificação das pesquisas, o que comprometeu a análise do fluxo através dos referidos documentos.

No município de Fortaleza a AF é organizada pela Célula de Assistência Farmacêutica (CELAF), órgão da esfera municipal responsável pela gestão dos insumos para abastecimento da APS. Entretanto, todo o ciclo logístico, assim como a gestão de pessoas estão sob a responsabilidade de uma "Organização Social de Saúde", demonstrando uma configuração anômala da AF no nível primário da atenção. Partindo da premissa de que avaliar é entendido como o resultado de cada ato de trabalho no cotidiano dos serviços de saúde e nos territórios sanitários, necessita-se analisar as práticas de saúde, a dimensão do alcance das ações e o contexto em que estão inseridos os sujeitos.

Assim, o passo seguinte foi entrevistar os profissionais farmacêuticos acerca da percepção deles sobre as pesquisas desenvolvidas na AF e a utilização dos seus resultados em suas práticas cotidianas: 
"Eu não conheço muitas não, tem o meu trabalho de TCC sobre farmácia comunitária. Já vi outros trabalhos que tive oportunidade de acompanhar mas não na APS, foi mais na farmácia clínica, outra com pacientes portadores de HIV, outro na UTI neonatal [...]".

Avaliar as pesquisas em saúde realizadas numa determinada área de estudos, pode revelar dimensões significativas para a gestão de recursos, de tempo, de demandas, de otimização de fluxos e de aplicabilidade dos resultados para a transformação das práticas na perspectiva da melhoria da saúde em geral.

No cotidiano desses profissionais, a tomada de decisão geralmente é baseada na experiência prévia de trabalho, em cursos de formação e na opinião de outros componentes da equipe. Portanto, não é comum, a utilização de resultados de pesquisas nesse processo conforme o discurso a seguir:

"Utilizo (os resultados de pesquisa), mas confesso que faço isso porque também estou no meio acadêmico. Para mim parece ser mais comum. Vejo essas coisas acontecendo no grupo de pesquisa. Aprendi a ser mais crítica. Nem sempre fui assim. Associar o serviço à academia é fundamental."

Os farmacêuticos afirmam que geralmente não há aplicabilidade ou devolutiva das pesquisas para os gestores, para os serviços e para a população:

"A pesquisa que Ihe falei (sobre pacientes com HIV) foi bastante longa, teve bons resultados, mas não houve continuidade após o término, quando terminou, já parou [...]".

"Os resultados não tiveram absorção nos serviços, não que eu saiba [...]".

"Teve uma pesquisa sobre transplante, que virou rotina no serviço, mas na APS mesmo, eu não conheço".

Ao se argumentar sobre os desafios percebidos para a implementação dos resultados das pesquisas em sua práxis e quais estratégias poderiam ser pensadas para facilitar sua utilização, os farmacêuticos relatam:

"Lógico que isso só é possível se a gestão tiver interesse naquele assunto. Se não for assim, esqueça! [...] Outra coisa fundamental é a equipe estar motivada. Houve um trabalho sobre o cuidado a pacientes hipertensos e diabéticos com farmacêuticos do NASF que foi um fracasso simplesmente porque não houve motivação da equipe".

"Por isso, que quando fui chamada para falar sobre o GAM (Gestão Autônoma da Medicação) numa reunião da equipe fiz a mais do que me pediram. Procurei a universidade, fiz parceria, desenvolvimento de curso, convidamos quem de fato está a fim de trabalhar com isso. Vai dar certo? Não sei! Mas estamos tentando o melhor caminho".

As complexidades seriam um excelente texto suplementar em cursos de graduação ou pós-graduação de divisão superior em lógica, filosofia, ciência política, gestão global de recursos humanos ou diversidade cultural. A natureza do mundo real dos ensaios e a falta de conclusões claras tornam as seleções neste livro um excelente ponto de partida para discussões em aula e exercícios de prática reflexiva. Usado efetivamente, este livro deve ajudar os alunos a demonstrar competência na síntese, avaliação e julgamento das vantagens e desvantagens dos cursos de comportamento.

Em meio às falas proferidas por esses participantes atuantes na APS de Fortaleza, buscou-se entender a estruturação da AF de Fortaleza e a distribuição dos farmacêuticos no território, no intuito de visualizar a aproximação deles com a APS.

A CELAF é o órgão municipal organizador da AF em Fortaleza e é responsável pelo gerenciamento dos insumos estratégicos oriundos do Ministério da Saúde e medicamentos de alto custo de vários programas, como: DST/AIDS, Saúde da Mulher, Nutrição e Alimentação, Tuberculose, Hanseníase, os insumos para crianças de baixo peso, além de outros insumos oriundos do Estado, como as insulinas. Além desses, a CELAF também é responsável pelos medicamentos de demanda judicial, planejamento de ações da educação permanente, dentre outras funções. $O$ fluxo desses insumos para a APS, passa pela Célula de Atenção Básica e é distribuída pelo Instituto de Saúde e Gestão Hospitalar (ISGH), o qual é uma instituição privada autoreferida como "sem fins lucrativos" que atua no segmento de gestão em saúde.

Ainda em campo, os profissionais revelaram que existem 93 UAPS e 13 CAPS (Centro de Atenção Psicossocial) sendo que em cada Regional de Saúde de Fortaleza havia um ou mais farmacêuticos, de modo que havia um farmacêutico clínico em cada CAPS e outro responsável pelo ciclo logístico, porém vinculado ao ISGH e não à CELAF. 
Na APS não havia um farmacêutico específico para as UAPS, porém, alguns ocupavam cargo de coordenação ou cargo técnico da regional, na ocasião em que foram abordados para a entrevista.

No momento da pesquisa, a CELAF estava coordenando um GT (Grupo de Trabalho) sobre cuidados clínicos farmacêuticos na Saúde Mental, entretanto foram realizadas várias tentativas de entrevistas com a gestora responsável por esse projeto, sem sucesso. Vale ressaltar que a contratação de farmacêuticos e a aquisição de medicamentos comprados pelo município, não são mais de responsabilidade da CELAF, mas do ISGH, muito criticada pelos farmacêuticos entrevistados, principalmente por realizar uma forma de contratação sem vínculos empregatícios, e pelo ineficiente abastecimento de alguns insumos, conforme o relato:

"Antes do ISGH, a comunicação circulava mais facilmente [...] Qualquer dúvida entrávamos em contato diretamente com a regional ou com a própria CELAF. Mas agora, o ISGH é responsável pela distribuição desses insumos para as UAPS e outros serviços [...] só que acontece de forma muito desorganizada, alguns recebem demais, outros ficam sem o medicamento. As pessoas não se empenham em preencher as informações corretamente e acaba dando nisso".

Quanto às funções que realizavam no momento da pesquisa, os farmacêuticos vinculados à APS abordaram atividades relacionadas ao medicamento, uma vez que suas ações estavam relacionadas à logística de insumos farmacêuticos, distante do cuidado integral requerido pelas novas políticas de reorientação farmacêutica.

A práxis do farmacêutico na APS estão engessadas em atividades individualizadas, burocráticas, à margem de práticas educativas que visem a prevenção e a promoção de saúde da comunidade. Porém, alguns discursos demonstram uma melhor articulação com outros profissionais e com práticas de educação permanente:

"Eu realizo mais atividades burocráticas: mapas, registro e dispensação nos livros de controle especial [...]". "Hoje exerço trabalhos gerenciais, ciclo da AF, especificamente referente a aquisição, distribuição [...] treinamentos, e o que mais gosto, o cuidado [...]. Não uso técnicas da atenção farmacêutica, mas oriento e registro tudo em prontuário. Qualquer dúvida entro em contato com os outros integrantes da equipe".

No território, percebe-se que a organização e a estruturação da AF em Fortaleza têm priorizado a execução de ações municipais, por meio de atividades restritas aos componentes técnicos ligado às etapas de seleção, programação, aquisição, armazenamento e distribuição de medicamentos. Ainda que a atividade de gestão contemple mais ações que a aplicação de técnicas específicas, a compressão da gestão da AF local se consolidou, na prática, como um conjunto de tarefas tecnicamente definidas e limitadas no âmbito das tecnologias duras das ciências farmacêuticas.

Não se deve desconsiderar a importância do farmacêutico nesse processo, porém esse profissional deve buscar ampliar seu horizonte de ações para alcançar excelência em seus serviços e a consolidação práticas que visem uma atenção integral. No campo empírico, os farmacêuticos reconhecem essa importância e apontam as dificuldades em consolidá-la:

"Eu acredito que o farmacêutico deveria andar mais junto do médico. O farmacêutico adora dizer que médico não aceita opinião de farmacêutico, mas isso não é verdade, eles adoram [...], você chega, faz a intervenção, questiona ou pergunta para ele [...]. Mas parece que o farmacêutico tem medo de sair daquela porta da farmácia, é o que eu sinto [...]. A integração da enfermagem, médico, farmacêutico, auxiliar de enfermagem [...] é o que deve ocorrer, mas parece que o farmacêutico não consegue, ele fica bitolado naqueles números de comprimidos, acha que a dispensação de medicamento é contar e entregar remédios e muitas vezes não se dá ao trabalho de perguntar ao paciente se ele sabe como tomar ou para que serve aquele medicamento".

"Para a gente ganhar espaço, a gente vai ter que se mostrar, não vai ser uma varinha de condão que vai fazer isso, temos que nos mostrar necessários [...]".

"A gente tem que conhecer o paciente e isso é possível, o paciente sempre está ali na farmácia, alguns vão mais de uma vez no mês [...] mas pra isso é preciso que o farmacêutico interaja com ele, converse, pergunte".

O contexto da APS no processo prático de cuidar, constitui um espaço de reflexão ação, fundado em saberes técnico-científicos e populares, culturalmente significativos para o exercício democrático. Atuar nesse âmbito pode provocar mudanças individuais e capacitação para atuar na família e na comunidade. Entretanto, no contexto estudado, as práticas do farmacêutico na APS ainda são pontuais e justificadas sob o ponto de vista da legislação que exige a presença do profissional para a dispensação de medicamentos de controle especial. Também, o modelo praticado no município de Fortaleza no ano pesquisado, pautado na terceirização do vínculo empregatício 
e da gestão da farmácia, tem provocado o isolamento da AF aos demais serviços e setores da APS, favorecendo a quebra da integralidade do cuidado e a perdas significativas na resolubilidade.

Diante dessas considerações, percebe-se que a prática farmacêutica precisa ser uniformizada e incorporada como um componente essencial nas Políticas Nacionais de Saúde e em seus marcos regulatórios, tornando-se urgente sua atualização e adaptação. $O$ avanço dos serviços farmacêuticos na APS deve ter origem no redesenho do processo de trabalho dos farmacêuticos, de modo a aprimorar atividades já existentes e a implementar novas atividades, entre as quais a clínica farmacêutica e as ações técnico-pedagógicas.

\section{DISCUSSÃO}

A AF exerce um importante papel na APS. A disponibilidade dos medicamentos na APS deve atender as necessidades epidemiológicas, com suficiência, regularidade e qualidade apropriadas, de forma integrada com uma orientação para o seu uso racional por meio de diferentes serviços ofertados no território ${ }^{(27)}$. Tais ações, se aplicam na interação do farmacêutico com os demais integrantes dos serviços, podendo ser intensificado no apoio ao prescritor e à grupos de usuários de risco ou polimedicados.

Quando se trata dos resultados de uma avaliação, é preciso levar em consideração que tais resultados são subsídios que podem influenciar a tomada de decisão do gestor, e devem ter o potencial de direcionar para uma mudança. Ao fazer uma avaliação, é importante que o farmacêutico faça o seguinte questionamento: Onde se quer chegar? Qual o resultado esperado da AF? Ter clareza do objetivo e da ação é um dos passos para alcançar os resultados desejados. A meta de um serviço deve estar direcionada pelo planejamento e, quando necessário, orientada por uma avaliação. Esta exige a emissão de um juízo de valor e só tem sentido com o direcionamento de um propósito, ou seja, com a finalidade da ação(5).

A ampliação da avaliação de políticas sociais no Brasil está direcionada à necessidade de acompanhamento dos investimentos realizados por financiadores e órgãos internacionais ${ }^{(2)}$. $\mathrm{O}$ fomento à avaliação é justificada pela busca de melhoria da gestão pública e prestação de contas aos gestores e à população.

Ressalta-se que a avaliação proporciona ao gestor, inclusive na APS, um grande escopo de informação que deve ser apresentada em tempo hábil, e deve se organizar de modo consistente, levando em conta o tempo e os recursos disponíveis. Porém, também se ressalta a disponibilidade de muitas bases de dados no serviço público, as quais, muitas vezes, não são utilizadas no cotidiano dos serviços para o planejamento e a avaliação das práticas desenvolvidas ${ }^{(28)}$.

O conhecimento no campo do cuidado só se torna possível se conseguir construir a pesquisa junto com os trabalhadores no cotidiano com produção intensiva do conhecimento no ato do cuidado ${ }^{(29)}$. É necessário, então, considerar o "cuidado" numa dimensão mais ampla e incorporando as questões sociais inerentes ao indivíduo e à coletividade. Uma pesquisa revela que o país conta com massa crítica e capacidade instalada de pesquisa em saúde ${ }^{(30)}$. No entanto, o Ministério da Saúde ainda não ocupou uma posição de predominância na definição de prioridades nacionais de pesquisa em saúde apesar de ter havido importante esforço nesse sentido, com medidas institucionais e financeiras, criação da Agenda Nacional de Prioridades de Pesquisa em Saúde que fosse capaz de integrar os diversos setores de interesse, a exemplo do Ministério da Ciência, Tecnologia e Inovação (MCT\&I).

Assim, são necessárias medidas que favoreçam a disseminação dos resultados das pesquisas para gestores e trabalhadores, no sentido de que as mesmas sejam devolvidas e aplicadas no cotidiano dessas pessoas e possam impactar na saúde e na qualidade de vida da população. Não só pesquisadores, mas os profissionais de saúde também devem desenvolver avaliações que forneçam informações para aprimorar os programas e os serviços de saúde, as quais devem ser dinâmicas e acompanhar as frequentes alterações dos objetos avaliados, tanto a nível estratégico quanto na prática cotidiana dos serviços ${ }^{(5)}$. A pesquisa em ato exige que um debate seja gerado entre toda a equipe sobre a própria finalidade do cuidado ${ }^{(31)}$, e a gestão não pode se eximir desta responsabilidade. Deveria ser da gestão o protagonismo do diálogo sobre qual o papel da pesquisa, levando em conta o espaço comum, relacional do trabalhador/gestor/usuário(32).

No Ceará, o ISGH iniciou suas atividades na administração do Hospital Dr. Waldemar Alcântara, e em 2011 passou a realizar a gestão do Hospital Regional do Cariri, e das quatro primeiras Unidades de Pronto Atendimento (UPAS 24h), em Fortaleza. Em 2013, foi iniciada a gestão do Hospital Regional Norte e de mais duas Unidades de Pronto Atendimento (UPAs). No mesmo ano, o Instituto foi contratado pela Prefeitura de Fortaleza, dando suporte operacional as atividades dos 93 postos de Saúde e dos $13 \mathrm{CAPS}^{(33)}$. A saúde no município de Fortaleza vem passando por um processo sutil de privatização do público, prática já recorrente na gestão pública brasileira, onde um montante dos gastos públicos na saúde tem direcionamento para o setor privado, em especial na forma de OSS, 
principalmente pela atuação na média e alta complexidade, entre elas os medicamentos e, mais recentemente, a gestão de pessoas ${ }^{(33)}$.

A lógica do modelo de terceirização implantado no município parece limitar o olhar do aspecto do medicamento apenas pela ótica econômica ou do planejamento, sob o risco de reduzir as demandas da população neste campo para uma circunstância anormal vivida pelo indivíduo, a doença ${ }^{(34)}$. Em conformidade com as políticas e a partir da demanda territorial da APS, o farmacêutico tem se aproximado da rede de serviços da APS, exercendo uma prática situada na transição de paradigmas entre o cuidado centrado no medicamento e o cuidado ou serviço farmacêutico 'ideal', que integra ações de educação em saúde, que incluem atividades de educação permanente para a equipe de saúde e atividades de promoção à saúde de caráter geral, além de ações de promoção do uso racional de medicamentos, com o desenvolvimento de atividades assistenciais e técnico-pedagógicas ${ }^{(27,35)}$.

Entre as atividades assistenciais que devem ser praticadas nos serviços de atenção, estão incluídos os serviços de clínica farmacêutica, que podem ser ofertados ao usuário de forma individual e/ou em atendimentos compartilhados com outros membros da equipe de saúde. As atividades técnico-pedagógicas, de forma complementar, visam à educação e ao empoderamento da equipe de saúde e da comunidade para a promoção do uso racional de medicamentos ${ }^{(36-38)}$.

A OPAS, ao descrever sobre os serviços farmacêuticos baseados na APS, direciona suas atribuições à atividades de educação e promoção da saúde e promoção do uso racional de medicamentos, denominando-as de atividades assistenciais e técnico-pedagógicas. As primeiras incluem os serviços de clínica farmacêutica, e as útlimas visam à educação e ao empoderamento da equipe de saúde e da comunidade para a promoção do uso racional de medicamentos ${ }^{(35)}$, bem como a adesão à um Plano Terapêutico Singular. Porém, na rotina dos serviços, as funções do farmacêutico devem se aproximar mais do foco proposto pela Política da Atenção Básica, as quais estão vinculadas, ao usuário, à sua família e à comunidade, ou seja, ao coletivo. A OPAS ${ }^{(35)}$ sugere que os serviços de clínica farmacêutica devem abranger um elenco de ações assistenciais nos pontos de atenção à saúde, ofertadas por meio de atendimento individual ou compartilhado com a equipe de saúde. Essas ações assistenciais podem incluir a dispensação de medicamentos, a orientação terapêutica ao usuário, o acompanhamento farmacoterapêutico, a revisão da farmacoterapia, a conciliação dos medicamentos e a avaliação e promoção da adesão terapêutica.

Pesquisas realizadas nos Estados Unidos e na Europa concluíram que as recomendações do farmacêutico melhoraram significativamente a adequação do uso dos medicamentos entre os usuários ${ }^{(15,39-41)}$. Além dessas atividades, é necessário organizar um sistema de referência e contrarreferência, entre os serviços de clínica farmacêutica da atenção básica e os serviços farmacêuticos clínicos ofertados nos níveis secundários e terciários. Assim, o farmacêutico em seu papel comunitário, deve estar integrado também à equipe, para esclarecer dúvidas sobre o medicamentos, podendo apoiar, inclusive, os prescritor quanto ao planejamento fármaco terapêutico do usuário ${ }^{(35)}$.

AAPS precisa garantir a presença do farmacêutico no cuidado integral ao paciente e fundamentar suas ações nas necessidades de saúde do indivíduo, da família e da comunidade numa perspectiva sistêmica já que a APS desenvolve ações intersetoriais com a promoção do cuidado em espaços de saúde, na educação e promoção de saúde. Desse modo, há urgência em se discutir um novo paradigma para a AF, sobretudo na APS, principalmente com relação a iniquidade do acesso aos medicamentos e a fragmentação dos processos que se observa no ponto de vista teórico das políticas farmacêuticas ${ }^{(42)}$.

\section{CONSIDERAÇÕES FINAIS}

A avaliação se revelou como prática incipiente e ainda não instituída na interface entre AF e APS. Este estudo aponta fragilidades quanto à utilização do conhecimento científico dos profissionais para a tomada de decisão e orientação de suas ações. Os farmacêuticos ainda não incorporaram o cuidado enquanto tecnologia de serviço farmacêutico na APS. Quanto às pesquisas existentes nesse âmbito, poucos reconhecem alguma aplicabilidade de seus resultados.

No território, os farmacêuticos (profissionais, pesquisadores, usuários e gestores) parecem estar perdidos quanto ao conhecimento científico produzido localmente, pois não há um fluxo eficaz desse produto, ou seja, as pesquisas realizadas nem sempre estão direcionadas à um problema existente no território e na maioria das vezes, os resultado gerados não são aplicados na prática. Isto também ocorre porque os profissionais não acessam a informação em tempo hábil e no formato viável à sua aplicabilidade, o que resulta em desperdício de tempo, recursos e trabalho.

O fluxo dessas pesquisas está interrompido, fragmentado, confuso e burocrático. Enquanto perspectiva futura, o presente trabalho sugere um melhor gerenciamento das pesquisas, propondo um fluxo efetivo e contínuo que tenha origem na real demanda da comunidade e cujos resultados sejam direcionados para a construção de novas estratégias que otimizem os recursos, as decisões e as práticas em saúde. 


\section{CONFLITOS DE INTERESSE}

Os autores declaram que não há conflito de interesses no referido estudo.

\section{REFERÊNCIAS}

1. Ministério da Saúde (BR). O que é Atenção Primária [Internet]? Brasília: Ministério da Saúde; [2018] [acesso em 2020 Fev 17]. Disponível em: http://dab.saude.gov.br/portaldab/dab.php

2. Furtado JPG, Gasparini MFV. Há diferenças entre avaliar e analisar? Ciênc Saúde Colet [Internet]. 2019 [acesso em 2020 Fev 17];24(8):2933-38. Disponível em: http://www.scielo.br/scielo.php?script=sci_ abstract\&pid=S1413-81232019000802933\&Ing=pt\&nrm=iso doi: 10.1590/1413-81232018248.25302017

3. Bezerra IC, Pinheiro CPO, Jorge MSB, Silva RM, Gonçalves JL, Mendonça FAC. Assistência farmacêutica na atenção primária à saúde: um estudo avaliativo. Anais do $8^{\circ}$ Congresso Ibero-Americano em Investigação Qualitativa; 2019 Jul 16-19; Lisboa. Aveiro (POR): CIAIQ; 2019.

4. Pena KS, Rollo RM, Reuter CLO, Santos VCF, Riquinho DL, Ramos AR. Care handover to chronic conditions to regionalized planning. Rev Gaúcha Enferm [Internet]. 2019 [acesso em 2020 Fev 17];41(spe):e20190168. Disponível em: http://www.scielo.br/scielo.php?script=sci_arttext\&pid=S1983-14472020000200406\&lng=en doi: 10.1590/1983-1447.2020.20190168

5. Conselho Federal de Farmácia (BR). O farmacêutico na assistência farmacêutica do SUS: diretrizes para ação. Brasília: Conselho Federal de Farmácia; 2015.

6. Organização Pan-Americana da Saúde; Organização Mundial da Saúde; Ministério da Saúde. Avaliação da assistência farmacêutica no Brasil: estrutura, processo e resultados. Ministério da Saúde. Brasília: Organização Pan-Americana da Saúde; 2005.

7. Bojke C, Philips Z, Sculpher M, Campion P, Chrystyn H, Coulton S, et al. Cost-effectiveness of shared pharmaceutical care for older patients: respect trial findings. Br J Gen Pract. 2010;60(570):20-7.

8. Rutter J, Fitzpatrick R, Rutter P. What effect does medicine advice provided by UK Medicines Information pharmacists have on prescriber practice and patient care: a qualitative primary care study. J Eval Clin Pract. 2015;21(2):307-12.

9. Richmond S, Morton V, Cross B, Wong ICK, Russell I, Philips Z, et al. Effectiveness of shared pharmaceutical care for older patients: respect trial findings. Br J Gen Pract. 2010;60(570):10-9.

10. Leendertse AJ, Koning GHP, Goudswaard AN, Belitser SV, Verhoef M, Gier HJ, et al. Preventing hospital admissions by reviewing medication (PHARM) in primary care: an open controlled study in an elderly population. J Clin Pharm Ther. 2013;38(5):379-87.

11. Guénette L, Maheu A, Vanier MC, Dugré N, Rouleau L, Lalonde L. Pharmacists practising in family medicine groups: What are their activities and needs? J Clin Pharm Ther. 2020;45:105-14.

12. Rose O, Schaffert C, Czarnecki K, Mennemann HS, Waltering I, Hamacher S, et al. Effect evaluation of an interprofessional medication therapy management approach for multimorbid patients in primary care: a cluster-randomized controlled trial in community care (WestGem study protocol). BMC Fam Pract. 2015;16.

13. Fiß T, Thyrian JR, Wucherer D, Aßmann G, Kilimann I, Teipel SJ, et al. Medication management for people with dementia in primary care: description of implementation in the DelpHi study. BMC Geriatr. 2013;13(1):121.

14. Leendertse AJ, Koning FHP, Goudswaard AN, Jonkhoff AR, van den Bogert SCA, Gier HJ, et al. Preventing hospital admissions by reviewing medication (PHARM) in primary care: design of the cluster randomised, controlled, multi-centre PHARM-study. BMC Health Serv Res. 2011;11:4.

15. Moullin JC, Sabater-Hernández D, García-Corpas JP, Kenny P, Benrimoj SI. Development and testing of two implementation tools to measure components of professional pharmacy service fidelity. J Eval Clin Pract. 2016;22(3):369-77.

16. Bona Sartor V, Freitas SFT. Modelo para avaliação do serviço de dispensação de medicamentos na atenção básica à saúde. Revista Saúde Pública. 2014;48(5):827-36. 
17. Menolli PVDS, Ivama AM, Cordoni L Jr. Description of the pharmaceutical services offered by the universal health care system at the primary care level in Londrina, Paraná, Brazil. Rev Panam Salud Pública. 2009;25(3):254-59.

18. Araujo ALA, Pereira LRL, Ueta JM, Freitas O. Perfil da assistência farmacêutica na atenção primária do Sistema Único de Saúde. Ciênc Saúde Colet. 2008;13(suppl.):611-7.

19. Vieira MRS, Lorandi $P A$, Bousquat $A$. Avaliação da assistência farmacêutica à gestante na rede básica de saúde do Município de Praia Grande, São Paulo, Brasil. Cad Saúde Pública. 2008;24(6):1419-28.

20. Serapione M. Conceitos e métodos para a avaliação de programas sociais e políticas públicas. Sociologia. 2016;31:59-80.

21. Patton MQ. Utilization-Focused Evaluation: the new century text. Thousand Oaks: Sage Publications; 1997.

22. Guba E, Lincoln Y. Fourth generation evaluation. Newbury Park: Sage Publications; 1989.

23. Habermas J. Dialética e Hermenêutica. Porto Alegre: L\&PM; 1987.

24. Minayo MCS. Hermenêutica: dialética como caminho do pensamento social. In: Minayo MCS, Deslandes SF, organizadores. Caminhos do pensamento: epistemologia e método. Rio de Janeiro: Editora FIOCRUZ; 2003. p. 83-107.

25. Minayo MCS, Costa AP. Fundamentos teóricos das técnicas de investigação qualitativa. Rev Lusófona Educ. 2018;40:139-53.

26. Assis MMA, Jorge MSB. Métodos de análise em pesquisa qualitativa. In: Santan JSS, Nascimento MAA, organizadores. Pesquisas, métodos e técnicas de conhecimento da realidade social. Feira de Santana: UEFS Ed; 2010. p. 139-59.

27. Ministério da Saúde (BR), Secretaria de Ciência, Tecnologia e Insumos Estratégicos, Departamento de Assistência Farmacêutica e Insumos Estratégicos. Serviços farmacêuticos na atenção básica à saúde. Brasília: Ministério da Saúde; 2014.

28. Tanaka OY, Tamaki EM. O papel da avaliação para a tomada de decisão na gestão de serviços de saúde. Ciênc Saúde Colet. 2012;17(4):821-8.

29. Gomes MPC, Merhy EE, Silva E, Abrahão AL, Vianna L, Rocha M, et al. Uma pesquisa e seus encontros: a fabricação de intercessores e o conhecimento como produção. In: Gomes MPC, Merhy EE, organizadores. Pesquisadores IN-MUNDO: um estudo da produção do acesso e barreira em saúde mental. Porto Alegre: Rede UNIDA; 2014. p. 25-42.

30. Guimarães R. Ciência, tecnologia e inovação: um paradoxo na Reforma Sanitária. In: Lima NT, Gerschman S, Edler FC, Suárez JM, organizadores. Saúde e democracia, história e perspectivas do SUS. Rio de Janeiro: Editora Fiocruz; 2005. p. 235-56.

31. Gomes MPC, Cipriano RC, Freire JT, Merhy EE, Silva E, Abrahão AL, et al. Acesso às multiplicidades do cuidado como enfrentamento das barreiras em saúde mental: Histórias de R. In: Gomes MPC, Merhy EL, organizadores. Pesquisadores IN-MUNDO: um estudo da produção do acesso e barreira em saúde mental. Porto Alegre: Rede UNIDA; 2014. p. 55-87.

32. Schiffler ÂCR, Abrahão AL. Interferindo nos microprocessos de cuidar em saúde mental. In: Gomes MPC, Merhy EE, organizadores. Pesquisadores IN-MUNDO: um estudo da produção do acesso e barreira em saúde mental. Porto Alegre: Rede UNIDA, 2014. p. 89-103.

33. Paim JS, Teixeira C.F. Política, planejamento e gestão em saúde: balanço do estado da arte. Rev Saúde Pública. 2006;40(spe):73-8.

34. Pinheiro R, Guizardi FL, Machado FRS, Gomes RS. Demanda em saúde e direito à saúde: liberdade ou necessidade? Algumas considerações sobre os nexos constituintes das práticas de integralidade. Rio de Janeiro: UERJ; [2013].

35. Organización Panamericana de la Salud. Servicios farmacéuticos basados en la atención primaria de salud. Washington, DC: OPAS; 2013. 
36. Leal LF, Bertoldi AD, Menezes AMB, Borges RB, Mengue SS, Gazzana MB, et al. Indicação, acesso e utilização de medicamentos para doenças respiratórias crônicas no Brasil: resultados da Pesquisa Nacional sobre Acesso, Utilização e Promoção do Uso Racional de Medicamentos no Brasil (PNAUM), 2014. Cad Saúde Pública [Internet]. 2018 [acesso em 2020 Fev 17];34(10):e00208217. Disponível em: https://doi. org/10.1590/0102-311X00208217 doi: 10.1590/0102-311X00208217

37. Organização Mundial de Saúde. Relatório Mundial da Saúde 2008: Cuidados de Saúde Primário - Agora mais que nunca. Genebra: OMS; 2008.

38. Ministério da Saúde (BR). Portaria n 198/GM/MS, de 13 de fevereiro de 2004. Institui a Política Nacional de Educação Permanente em Saúde como estratégia do Sistema Único de Saúde para a formação e o desenvolvimento de trabalhadores para o setor e dá outras providências. Brasília: Ministério da Saúde; 2004.

39. Benedict AW, Spence MM, Sie JL, Chin HA, Ngo CD, Salmingo JF, et al. Evaluation of a pharmacist-managed diabetes program in a primary care setting within an integrated health care system. J Manag Care Spec Pharm. 2018;24(2):114-22.

40. Chen J, Sena K, Nawarskas AD, Smith CA, Ward K. Impact of pharmacy medication reviews on the prescribing of potentially inappropriate medications for older veterans in primary care. Sr Care Pharm. 2019;34(7):464-72.

41. Jeong S, Lee M, Ji E. Effect of pharmaceutical care interventions on glycemic control in patients with diabetes: a systematic review and meta-analysis. Ther Clin Risk Manag. 2018;14:1813-29.

42. Barberato LC, Scherer MDA, Lacourt RMC. O farmacêutico na atenção primária no Brasil: uma inserção em construção. Ciênc Saúde Colet. 2019;24(10):3717-26.

\section{Endereço para correspondência:}

Indara Cavalcante Bezerra

Universidade Estadual do Ceará

Av. Silas Munguba, 1700

Bairro: Itaperi

CEP: 60714-903 - Fortaleza - CE - Brasil

E-mail: indaracavalcante@yahoo.com.br 